\title{
LACK OF MONOTONICITY IN SPIN GLASS CORRELATION FUNCTIONS
}

\author{
Pierluigi Contucci $^{\dagger}, \quad$ Francesco Unguendoli ${ }^{\ddagger}, \quad$ Cecilia Vernia ${ }^{\ddagger}$, \\ † Dipartimento di Matematica, Università di Bologna, e-mail: contucci@dm.unibo.it \\ $\ddagger$ Dipartimento di Matematica, Università Modena, e-mail: unguendoli@unimore.it, \\ cecilia.vernia@unimore.it
}

\begin{abstract}
We study the response of a spin glass system with respect to the rescaling of its interaction random variables and investigate numerically the behaviour of the correlation functions with respect to the volume. While for a ferromagnet the local energy correlation functions increase monotonically with the scale and, by consequence, with respect to the volume of the system we find that in a general spin glass model those monotonicities are violated.
\end{abstract}

When spins interact in a ferromagnetic system they tend to be aligned. That simple fact is reflected in structural properties of the statistical mechanics equilibrium state called Griffiths inequalities [Gr, Gr2] or more generally GKS [KS] inequalities.

The first inequality states that the pressure of a spin system (minus the free energy times beta) does increase with the strength of each interaction among spins. The second says that the correlation among any set of spins increases with respect to the strength of the interaction of any other set of them.

Since the strength of the interaction allows to switch on and off new parts of the system, the mentioned monotonicity properties can be easily turned into new ones like monotonicity with respect to the volume or with respect to the system dimensionality. 
All those properties are at the origins of the fruitful applications of those inequalities to prove rigorous results in statistical mechanics, like the existence of the thermodynamic limit for pressure and correlations, bounds for critical temperatures and exponents [BS] and their mutual relations for different systems.

While the physical meaning of the Griffiths inequalities was clear and well understood much earlier than rigorously proved, for disordered systems like spin glasses there are no $a$ priori evident monotonicity properties due to the lack of ferromagnetism and the presence of competition (frustrated loops [MPV]).

The first case to be understood has been the Gaussian interaction. The use of the partial integration led to the first general proof of existence and monotonicity of thermodynamic limit for the pressure of a d-dimensional spin glass model with general potential $[\mathrm{CG}]$. Defining the potential

$$
U_{\Lambda}(J, \sigma)=\sum_{X \subset \Lambda} J_{X} \sigma_{X}
$$

where the coefficients $J_{X}$ are a Gaussian family distributed as

$$
\operatorname{Av}\left(J_{X}\right)=0, \quad \operatorname{Av}\left(J_{X} J_{Y}\right)=\Delta_{X}^{2} \delta_{X, Y}
$$

with its associated random Gibbs-Boltzmann state $\omega$ and the quenched measure as

$$
<->=\operatorname{Av}[\omega(-)]
$$

a straightforward computation $[\mathrm{CG}]$ gives

$$
<J_{X} \sigma_{X}>=\Delta_{X}^{2} \operatorname{Av}\left(1-\omega_{X}^{2}\right)
$$

which turns out to be positive by inspection. Although it was clear that the former positivity comes from convexity, it was recognized only few years later that the same result holds in full generality for random interaction $J_{X}$ with zero average and not only for centered Gaussian variables: in [CL] it is proved that (0.4) can be simply derived from thermodynamic convexity.

A natural perspective to look at the former inequalities is to consider the deformation of the general centered random variable $J_{X}$ as $\lambda_{X} J_{X}$ with $\lambda_{X}>0$. The quenched pressure $P$ as a function of the set of lambda's has first and second derivatives 


$$
\begin{gathered}
\frac{\partial P}{\partial \lambda_{X}}=<J_{X} \sigma_{X}>\geq 0 \\
\frac{\partial^{2} P}{\partial \lambda_{X} \partial \lambda_{Y}}=\frac{\partial<J_{X} \sigma_{X}>}{\partial \lambda_{Y}}=\operatorname{Av}\left[J_{X} J_{Y}\left(\omega_{X Y}-\omega_{X} \omega_{Y}\right)\right]
\end{gathered}
$$

The two quantities (0.5) and (0.6) have been extensively studied in $d=1$ with nearest neighboor interaction and periodic (or free) boundary conditions in [CU]: the sign of (0.5) remains positive also by shifting on positive values the $J$ averages; the value of (0.6) turns out to be non positive for zero mean and positive variance interactions and changes its sign when crossing a line in the mean-variance plane toward the ferromagnetic regime of zero variance and positive mean.

The present paper deals with the study of the sign of (0.6) for higher dimensions or different topologies for the case with zero average interaction. Our findings can be summarized as follows: (0.6) doesn't have a definite sign. An explicit counterexample is found, for instance, in the case of a nearest neighboor spin chain with an extra interaction connecting two distant spins. In principle, a specific topology could not affect the monotonicity in the volume. For that reason we test numerically the nearest neighboor correlation function for two and three dimensional systems of increasing size and find an oscillating behaviour.

Let consider a closed chain of six spins with nearest neighboor interaction with one added interaction between the spins 2 and 5 . The dependence of the partition function on the couplings $J_{1,2}$ e $J_{2,3}$ is:

$$
\begin{aligned}
Z= & \sum_{\sigma} \exp \left(\beta \sum J_{i, j} \sigma_{i} \sigma_{j}\right)= \\
= & a \cosh \left(\beta J_{1,2}\right) \cosh \left(\beta J_{2,3}\right)+b \sinh \left(\beta J_{1,2}\right) \cosh \left(\beta J_{2,3}\right)+ \\
& +c \cosh \left(\beta J_{1,2}\right) \sinh \left(\beta J_{2,3}\right)+d \sinh \left(\beta J_{1,2}\right) \sinh \left(\beta J_{2,3}\right)
\end{aligned}
$$

where the four coefficients are:

$$
a=\left(\prod_{(i, j) \neq(1,2),(2,3)} \cosh \left(\beta J_{i, j}\right)\right) \cdot \sum_{\partial B=\emptyset}\left(\prod_{(i, j) \in B} \tanh \left(\beta J_{i, j}\right)\right)
$$




$$
\begin{aligned}
& b=\left(\prod_{(i, j) \neq(1,2),(2,3)} \cosh \left(\beta J_{i, j}\right)\right) \cdot \sum_{\partial B=(1,2)}\left(\prod_{(i, j) \in B} \tanh \left(\beta J_{i, j}\right)\right) \\
& c=\left(\prod_{(i, j) \neq(1,2),(2,3)} \cosh \left(\beta J_{i, j}\right)\right) \cdot \sum_{\partial B=(2,3)}\left(\prod_{(i, j) \in B} \tanh \left(\beta J_{i, j}\right)\right) \\
& d=\left(\prod_{(i, j) \neq(1,2),(2,3)} \cosh \left(\beta J_{i, j}\right)\right) \cdot \sum_{\partial B=(1,2) \cup(2,3)}\left(\prod_{(i, j) \in B} \tanh \left(\beta J_{i, j}\right)\right)
\end{aligned}
$$

The truncated correlation function is now:

$$
\omega_{12,23}-\omega_{12} \omega_{23}=16 \frac{a d-b c}{Z^{2}}
$$

By Gauge invariance the Bernoulli random model can be reduced to one in which the randomness is concentrated on the two couplings $J_{1,2}= \pm 1, J_{2,3}= \pm 1$ with probability $1 / 2$, and the remaining others $J_{i, j}=1$.

Let introduce the notation: $C:=\cosh (\beta), S:=\sinh (\beta), T:=\tanh (\beta)$, and: $T_{12}:=$ $\tanh \left(\beta J_{1,2}\right), T_{23}:=\tanh \left(\beta J_{2,3}\right)$ (and similarly for sinh and $\cosh$ ).

We will indicate with $Z(+,+), Z(+,-), Z(-,+)$ and $Z(-,-)$ the partition functions computed with fixed values of $J_{1,2}$ e $J_{2,3}$.

An explicit computation gives:

$$
\begin{gathered}
a=C^{5} ; \quad b=c=C^{5} T^{3} ; \quad d=C^{5} T^{4} \quad \Rightarrow \\
\Rightarrow a d-b c=C^{10} T^{4}\left(1-T^{2}\right)
\end{gathered}
$$

and

$$
\begin{aligned}
Z=C^{5} C_{1} C_{2}\left[1+T_{12} T^{3}+\right. & \left.T_{23} T^{3}+T_{12} T_{23} T^{4}\right] \Rightarrow \\
Z(++) & =C^{7}\left[1+2 T^{4}+T^{6}\right] \\
Z(+-)=Z(-+) & =C^{7}\left[1-T^{6}\right] \\
Z(--) & =C^{7}\left[1-2 T^{4}+T^{6}\right]
\end{aligned}
$$


Finally:

$$
\begin{aligned}
& \operatorname{Av}\left[J_{1,2} J_{2,3}\left(\omega_{12,23}-\omega_{12} \omega_{23}\right)\right]=16 C^{10} T^{4}\left(1-T^{2}\right) \operatorname{Av}\left[\frac{J_{1,2} J_{2,3}}{Z^{2}}\right]= \\
& \quad=16 C^{10} T^{4}\left(1-T^{2}\right)\left\{\frac{1}{Z^{2}(++)}+\frac{1}{Z^{2}(--)}-\frac{2}{Z^{2}(+-)}\right\}= \\
& =\frac{16 T^{4}\left(1-T^{2}\right)}{C^{4}}\left\{\frac{1}{\left(1+2 T^{4}+T^{6}\right)^{2}}+\frac{1}{\left(1-2 T^{4}+T^{6}\right)^{2}}-\frac{2}{\left(1-T^{6}\right)^{2}}\right\}= \\
& \quad=\frac{16 T^{4}\left(1-T^{2}\right)}{C^{4}} \frac{8 T^{6}\left[-1+3 T^{2}-2 T^{6}+2 T^{8}-4 T^{10}-T^{12}+3 T^{14}\right]}{\left(1+2 T^{4}+T^{6}\right)^{2} \cdot\left(1-2 T^{4}+T^{6}\right)^{2} \cdot\left(1-T^{6}\right)^{2}}
\end{aligned}
$$

In order to compute the sign, we notice that the square parenthesis term is:

$$
\begin{aligned}
& {\left[-1+3 T^{2}-2 T^{6}+2 T^{8}-4 T^{10}-T^{12}+3 T^{14}\right]=} \\
& \quad=\left[\frac{C^{2}\left(S^{2}-1\right)\left(C^{8}-S^{8}\right)+2 S^{4}\left(C^{8}-S^{8}\right)+S^{8}\left(C^{2}+S^{2}\right)}{C^{14}}\right]
\end{aligned}
$$

The only term with possible sign change is $\left(S^{2}-1\right)$. For small $\beta$ the leading term is then $-\cosh ^{10}(\beta)$, which gives a negative contribution, while for large $\beta$ everything is positive. A plot of the function $\operatorname{Av}\left[J_{1,2} J_{2,3}\left(\omega_{12,23}-\omega_{12} \omega_{23}\right)\right]$ shows a change of sign around $\beta=0.695$.

The numerical test is performed for $d$-dimensional cubic lattices $\Lambda$ of volume $N=L^{d}$, with $d=2,3$. We analyze two cases of quenched disorder: the Bernoulli couplings with $J_{i, j}= \pm 1$ and the Gaussian couplings with zero mean and unit variance.

Given a spin configuration $\sigma$ for a system of linear size $L$, we consider the observable:

$$
A v\left[J_{b} \omega_{b}\right]
$$

where $b=(i, j)$ with $i, j \in \Lambda,|i-j|=1, \omega_{b}$ is the thermal average of the quantity $\sigma_{i} \sigma_{j}$ and $A v[\cdot]$ is the average over the quenched disorder.

With a parallel-tempering algorithm $[\mathrm{HN}]$ we investigate the correlation (0.7) for lattice sizes ranging from $L=3$ to $L=24$ in the case $d=2$ and from $L=3$ to $L=10$ 


\begin{tabular}{|c|c|c|c|c|c|c|c|}
\hline \multicolumn{7}{|c|}{ Two dimensional lattice $L^{d}, d=2$} \\
\hline$L$ & Therm & Equil & Nreal & $n_{t}$ & $\delta t$ & $t_{\text {min }}$ & $t_{\text {max }}$ \\
\hline \hline $3-12$ & 50000 & 50000 & 4096 & 19 & 0.1 & 0.5 & 2.3 \\
\hline 16 & 50000 & 50000 & 2048 & 19 & 0.05 & 0.5 & 2.3 \\
\hline 24 & 50000 & 50000 & 2600 & 19 & 0.05 & 0.5 & 2.3 \\
\hline \hline \multicolumn{7}{|c|}{ Three dimensional lattice $L^{d}, d=3$} \\
\hline$L$ & Therm & Equil & Nreal & $n_{t}$ & $\delta t$ & $t_{\text {min }}$ & $t_{\text {max }}$ \\
\hline \hline $3-6$ & 50000 & 50000 & 2048 & 19 & 0.1 & 0.5 & 2.3 \\
\hline 8 & 50000 & 50000 & 2680 & 19 & 0.1 & 0.5 & 2.3 \\
\hline 10 & 70000 & 70000 & 2048 & 37 & 0.05 & 0.5 & 2.3 \\
\hline
\end{tabular}

Table 1: Parameters of the simulations: linear system size, number of sweeps used for thermalization, number of sweeps for measurement of the observable, number of disorder realizations, number of temperature values allowed in the parallel tempering procedure, temperature increment, minimum and maximum temperature values.

in the case $d=3$. For each size we consider at least 2048 disorder realizations and, in order to thermalize the large sizes, we choose up to 37 temperature values in the range $0.5 \leq t \leq 2.3$, in which the critical temperatures of the three dimensional models $\left(t_{c} \simeq 0.95\right.$ for the Gaussian model [MPRL] and $t_{c} \simeq 1.15$ for the Bernoulli model $\left.[\mathrm{BCF}]\right)$ are contained. The thermalization in the parallel tempering procedure is tested by checking the symmetry of the probability distribution for the standard overlap $q$ under the transformation $q \rightarrow-q$. Moreover, for the Gaussian coupling case it is available another thermalization test: the internal energy can be calculated both as the temporal mean of the Hamiltonian or, using integration by parts, as the expectation of a simple function of the link overlap [Co]. We checked that with our thermalization steps both measurements converge to the same value. All the parameters used in the simulations are reported in Tab.1.

The numerical results are displayed in Fig.1 and in Fig.2, where the correlation (0.7) is 


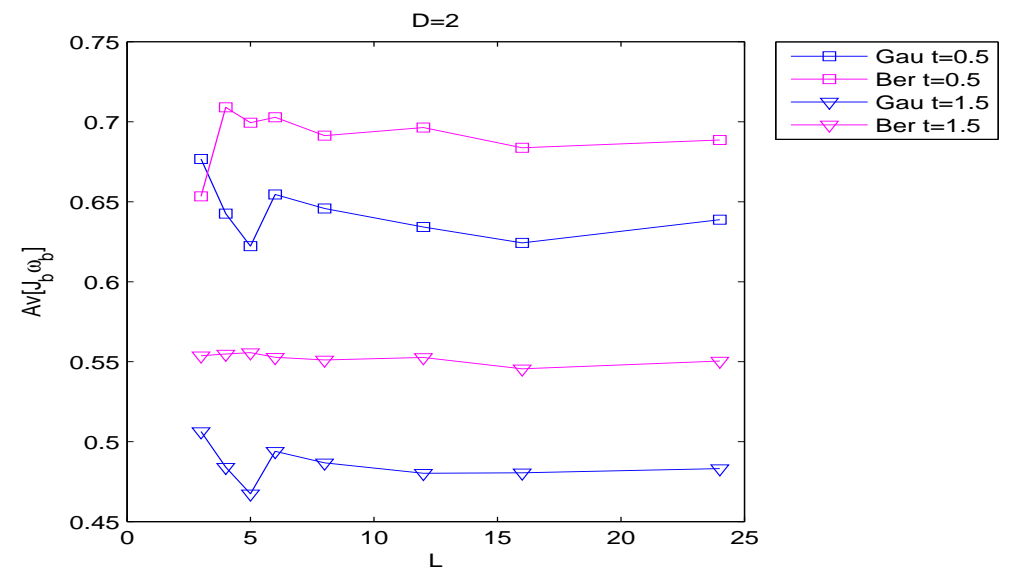

Figure 1: $A v\left[J_{b} \omega_{b}\right]$ as a function of $L$ for the two dimensional lattice with gaussian and Bernoulli coupling, for two different temperature values $t=0.5$ and $t=1.5$ each.

represented as a function of the linear system size $L$ for different temperatures both for the two dimensional system and for the three dimensional one. We find that the correlation oscillates with respect to $L$, independently of the couplings (Bernoulli and gaussian), of the temperatures and of the dimension $d(d=2,3)$.

The results presented here show that the monotonocity properties typical of ferromagnetic systems are clearly violated for spin glass models. Further effort is necessary to establish if the quantity (0.6) can keep a definite sign for specific lattice geometries or may depend on the relative position of the two sets $X$ and $Y$.

Acknowledgments. P.C. thanks Hal Tasaki for suggesting the topology used in analytical example. Cristian Giardiná, Sandro Graffi, Frank Den Hollander and Hidetoshi Nishimori are acknowledged for many useful discussions.

\section{References}

[CL] P.Contucci, J.Lebowitz, Correlation Inequalities for Spin Glasses, Annales Henri Poincare, Vol. 8, N. 8, 1461-1467, (2007)

[CG] P.Contucci, S.Graffi, Monotonicity and thermodynamic limit, J. Stat. Phys., Vol. 115, Nos. 1/2, 581-589, (2004) 


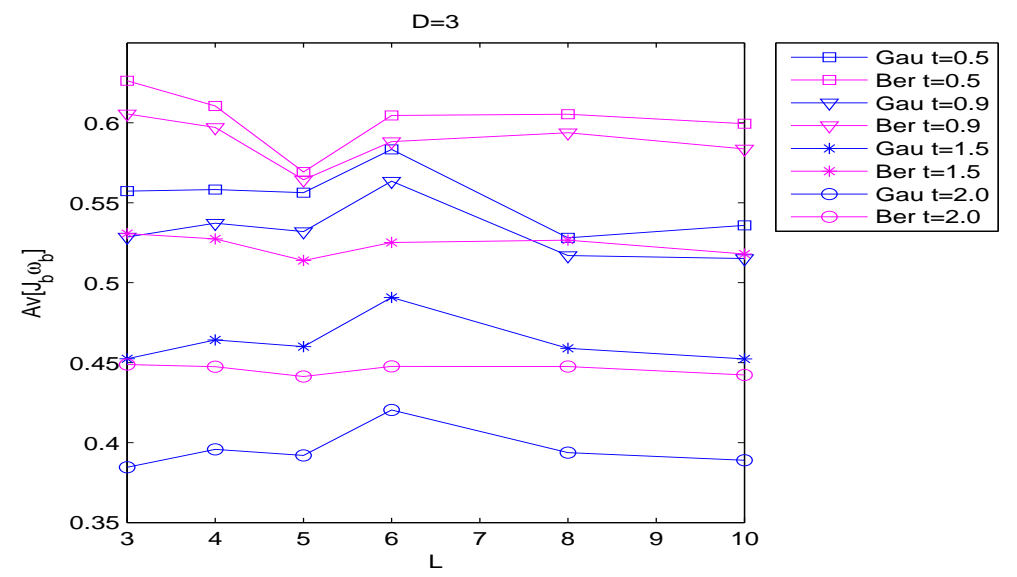

Figure 2: $A v\left[J_{b} \omega_{b}\right]$ as a function of $L$ for the three dimensional lattice with gaussian and Bernoulli coupling and for four different temperature values $t=0.5, t=0.9, t=1.5$ and $t=2$ each.

[Gr] R. B. Griffiths, Correlation in Ising Ferromagnets, J. Math. Phys., Vol. 8, 478-483, (1967)

[Gr2] R. B. Griffiths, A proof that the free energy of a spin system is extensive, J. Math. Phys., Vol. 5, 1215-1222, (1964)

[KS] D.G.Kelly, S. Sherman, General Griffiths' Inequalities on Correlations in Ising Ferromagnets, J. Math. Phys., Vol. 9, 466-484, (1968)

[BS] B. Simon, The Statistical Mechanics of Lattice Gases, Princeton University Press, (1993)

[CU] P. Contucci, F. Unguendoli, Correlation inequalities for spin glass in one dimension, Rend. Lincei Mat. Appl., to appear

[MPV] M. Mezard, G. Parisi, M. A. Virasoro, Spin Glass Theory and Beyond, World Scientific, (1987)

[CMN] P.Contucci, S.Morita, H.Nishimori, Surface Terms on the Nishimori Line of the Gaussian Edwards-Anderson Model, J. Stat. Phys., Vol. 122, N. 2, 303-312, (2006) 
[MNC] S.Morita, H.Nishimori, P.Contucci, Griffiths Inequalities for the Gaussian Spin Glass, J. Phys. A: Math. Gen., Vol. 37, L203-L209, (2004)

[KNA] H.Kitatani, H.Nishimori, A.Aoki, Inequalities for the local Energy of Random Ising Models, J. of the Physical Society of Japan, Vol. 76, Issue 7, pp. 074711 (2007).

[CG2] P.Contucci, S.Graffi, On the surface pressure for the Edwards-Anderson Model, Comm. Math. Phys. Stat. Phys., Vol. 248, 207-220, (2004)

[Co] P. Contucci, Replica Equivalence in the Edwards-Anderson Model, J. Phys. A: Math. Gen., Vol. 36, 10961-10966, (2003).

[MPRL] E. Marinari, G. Parisi, and J.J. Ruiz-Lorenzo, Phase structure of the threedimensional Edwards-Anderson spin glass, Phys. Rev. B, Vol. 58, 14852-14863 (1998)

[BCF] H.G. Ballesteros, A. Cruz, L.A. Fernandez, V. Martin-Mayor, J. Pech, J.J. RuizLorenzo, A. Tarancon, P. Tellez, C.L. Ullod, C. Ungil, Critical Behavior of the Three-Dimensional Ising Spin Glass, Phys. Rev. B, Vol. 62, 14237-14245 (2000)

[HN] K. Hukushima, K. Nemoto, Exchange Monte Carlo Method and Application to Spin Glass Simulations, J. of the Physical Society of Japan, Vol. 65, 1604-1608 (1996) 